\title{
Interactive comment on "Concentrations, Particle-Size Distributions, and Dry Deposition Fluxes of Aerosol Trace Elements over the Antarctic Peninsula" by Songyun Fan et al.
}

\section{Anonymous Referee \#2}

Received and published: 30 September 2020

This study investigated the concentrations, size distributions, and dry deposition fluxes of about 15 airborne trace elements in the Antarctic Peninsula. The results will be useful for the scientific community, especially considering the fact that the particulatebound elemental analysis for remote areas is scarce. However, there are scopes of improving the data analysis approach that will make the conclusions of the study more reliable.

General comments:

1. The total sampling period of the study is rather short ( $\sim 2$ months). Authors need to discuss the possible uncertainties/seasonal influence affecting the element concentra- 
tions, especially in the context of comparison with other studies.

2. The study used Al concentration ( $8 \%$ of the dust concentration) to calculate the dry and total deposition of dust in the region. A few major conclusions of the study were based on this assumption. The assumption could lead to uncertainties in the results as it is based on the Al concentration only and may not represent the crustal matter in the study region. Therefore, the authors should provide sufficient evidence to establish that this is a solid assumption. Mass reconstruction of soil/dust based on all available crustal elements concentration (rather than just the Al concentration) can also be considered.

3. Some figures and parts of the text should be revised to improve the clarity (e.g., Figures 4, Section 3.2.1, etc.). Specific line-by-line comments are as follows.

Specific comments:

Line 10: "The results show..." The reader would be wondering about the study methods/data analysis approach that leads to these results (i.e., enrichment calculation or statistical analysis/ source apportionment tool, etc.?).

Lines 11-13: "Elements dominated by a crustal source. . . reflecting the contributions of regional crustal sources" repetitive.

Line 13: The term "EFcrust" may not be familiar to all readers.

Line 13: ". . coarse-mode particles $(>1 \mu \mathrm{m})$. .." contradicts with lines $72-73$ that states "...those $>=1.8 \mu \mathrm{m}$ were summed to define coarse-mode particles. ..".

Lines 26-27: "It has been realized that the impact of coarse mineral dust has been underestimated. .." I am not sure what argument is presented here.

Lines 54-56: "However,... are lacking." Is this the first study on elements sampled in Antarctica? If yes, state clearly, if not, briefly mention about the prior studies that sampled aerosol in this region and what this study introduces. The paragraph that

Printer-friendly version

Discussion paper 
follows (lines 57-65) also does not give much idea about the knowledge gap that this study fills in.

Section 2, Line 66 onward: No mention of how the samples below "limits of detection (LOD)" were treated. Lines 77-78: "... wind direction inside the sector $\pm 60^{\circ}$ from the direction of the station and wind speed $<2 \mathrm{~m} \mathrm{~s}-1$." Unclear to me.

Line 91: “...Al, P, Ca, Ti, V, Mn, Ni, Cu, and Zn” this list looks different from the one mentioned in the abstract (line 9) that states "... Al, P, Ca, Ti, V, Mn, Ni, Cu, Zn, Ce, and $\mathrm{Pb} . . .$.

Line 133: The summation is over what variable?

Line 135: So, Vd was calculated from the combination of two models?

Line 161: "The values of EFcrust for Ti, V, Mn, and Ce in aerosol samples were less than 10..." Why $P$ is missing from this list where it has EFcrust $<10$ (Figure 3)?

Lines 170-171: "A similar phenomenon was observed at McMurdo Station where lightweight fuel oil was used that was not a significant source of V" not clear what was referred here.

Lines 173-185: My understanding is that the purpose of this paragraph is to establish that the enrichment of $P$ is higher than other crustal elements. But based on the results (Figure 3), it appears that $P$ enrichment is not significantly high in this study, compared to the other relevant studies.

Lines 197-198: "Hence, despite the recent increase in tourist ship traffic, it looks that Palmer Station was barely impacted by ship emissions" assuming this is correct, what is the reason for large variations of $\mathrm{Ni}$ (e.g., Figure 3$)$ ?

Lines 199-200: "Ca accounts for about 3.5\% of the weight of Earth's crust, while Ca is also a conservative major ion in seawater" provide citation.

Line 215: ". . .the impact of the nearby McMurdo Dry Valleys" I could not understand 
what impact was referred here.

Lines 217-218: "The concentrations of $\mathrm{Ti}$ and $\mathrm{Mn}$ ranged from. . respectively." I am a little confused if the ranges are for individual elements or both elements combined.

Lines 220-223: "...but comparable to the concentrations... (Figure $4 \mathrm{e}$ and g)" is it applicable to both $\mathrm{Ti}$ and $\mathrm{Mn}$ ? Figures $4 \mathrm{e}$ and $4 \mathrm{~g}$ do not support this claim. Mn concentration at AP-OS is more than double of AP-PS (Figure $4 \mathrm{~g}$ ).

Line 232: “. . .the $P$ values. .." $P$ concentrations?

Line 235-236: "Comparing global aerosol P concentrations. . as those over the Central Pacific Ocean (Chen, 2004)" provide the concentration values from the referred study.

Lines 236-237: "Confirming that Palmer Station was little influenced by aerosols derived from biomass burning through long-range transport, the calculated non-sea-salt$\mathrm{K}$ was indistinguishable from zero." I could not understand what this means.

Lines 239-241: “. . suggesting that aerosol crustal elements observed at Palmer Station were impacted by sources in that region (Figure 5)." Why this argument applies to only crustal elements?

Lines 263-264: "The low concentrations of heavy metals observed during this study suggest that local anthropogenic emissions were negligible." Which metals you are referring to?

Lines 264-265: "Thus the major source of non-crustal elements in aerosols over the study region may be long-range transport from regions impacted by anthropogenic emissions" very weak conclusion as it is only based on $\mathrm{Pb}$ variation.

Line 271: “. . .27 and 26, in seawater” respectively?

Lines 272-273: "The results suggest that Ca was dominated by sea-salt aerosol..." what about $\mathrm{K}$ ? 
Line 276: "classified into three groups based on their potential dominant sources" is this classification is in the context of section 3.1? If yes, it should be stated clearly. If that's not the case, provide justification of the grouping.

Lines 301-302: "The mass distributions of sea-salt elements ( $\mathrm{Ca}, \mathrm{Na}$ and $\mathrm{K})$ as the third group were dominated by coarse-mode particles with diameters 2.5-7.8 $\mu \mathrm{m}$ (Figure 6)" I could not find the size distributions of $\mathrm{Na}$ and $\mathrm{K}$ in Figure 6!

Interactive

comment

Line 303: "...the correlation between the total concentrations of $\mathrm{Ca}$ and $\mathrm{Na}$ was strongly positive (R2 \pm 0.82 , p-value $<0.01) \ldots$ " was it based on the 8 pairs of samples presented in Table 3? What about the correlations of other elements (such as K) with $\mathrm{Na}$ ? Like $\mathrm{Ca}$, If $\mathrm{K}$ is also associated with seasalt (as suggested in lines 270-272) one would expect a good K-Na correlation.

Lines 311-313: "The rough estimates of the dry deposition fluxes of $\mathrm{Ni}, \mathrm{Cu}$, and $\mathrm{Zn}$ at Palmer Station..." should mention few values from the literature so the readers get an idea of how large or small the values are.

Lines 313-315: "The estimated dry deposition fluxes of total continental dust. . among the lowest globally (Lawrence and Neff, 2009)" should mention a few global values.

Lines 316-319: “. . . precipitation scavenging accounted for about $40 \sim 60 \%$ of the total deposition..." are these fractions yearly average? Contributions of wet deposition to the total flux is a strong function of season. This study is limited to only two months of sampling. Authors need to discuss the likely uncertainties involved with extrapolating the short-term dry or wet deposition flux to yearly contributions.

Lines 318-319: "Assuming this wet deposition fraction applies to the Antarctic Peninsula region, we approximate roughly a total dust flux of $10 \mathrm{mg} \mathrm{m}-2 \mathrm{yr}-1$ " I understand that this is an estimation, but how was it obtained? Assuming dry/total as 0.4 ? or $0.6 ?$

Lines 329-341: Authors need to carefully revise this entire paragraph to ensure it is readable.

Printer-friendly version

Discussion paper 
Lines 346-348: "Most of the samples collected during this study were impacted by air masses originating around or passing over Northern Antarctic Peninsula..." This claim is not supported by any strong evidence. Airmass trajectories associated with 4 of the total samples were presented in Figure 5.

Lines 354-357: "As the role of wet deposition is unquantified at. . . may need to be reevaluated." I could not understand what this sentence is referring to.

Interactive

comment

Figure 2: Missing proper $x$-axis label. In addition, the figure caption should mention what the legends (M1...M10) are referring to.

Figure 4: This figure should be revised. 1) What is AP-OS1 shown in Figure 4(g)? 2) What are the vertical lines representing? 3) The acronyms of the sites mentioned in the caption should be consistent with the ones shown on figures (e.g., AP-PS or PS). 4) As mentioned in the text (e.g., line 211), air mass samples from many of the previous studies used for the comparison correspond to PM10 or even PM2.5. The figures should clearly indicate this (e.g., AP-OS(PM2.5)).

Figure 5: Why is this figure showing 4 samples only?

Table 4: Should include a few extra columns showing literature values.

Interactive comment on Atmos. Chem. Phys. Discuss., https://doi.org/10.5194/acp-2020-651, 2020. 